\title{
Cuidados paliativos oncológicos em um serviço público de atenção domiciliar
}

\author{
Oncology palliative care in a public home-care service \\ Cuidados paliativos oncologicos en un servicio publico de atención domiciliaria
}

Patrícia Pilattia ${ }^{a}$ Verlaine Balzan Lagnia ${ }^{a}$ Milene Campanholo Picasso ${ }^{b}$, Karen Puma ${ }^{b}$, Raquel Jeanty de Seixas Mestrinera, Diani de Oliveira Machadoa, Sati Jaber Mahmuda, Gislaine dos Santos Jardima , Heloisa Arrussul Bragaa , Carine Raquel Blatt Karine Zortéa ${ }^{a}$

\section{Resumo}

Objetivo: Identificar o perfil dos pacientes com câncer e em cuidados paliativos e suas demandas referentes ao atendimento multidisciplinar existente em um Serviço de Atenção Domiciliar. Métodos: Estudo transversal com coleta de dados secundários dos pacientes oncológicos em cuidados paliativos atendidos pelo Programa de Atenção Domiciliar do Grupo Hospitalar Conceição, no ano de 2013. Aprovado pelo Comitê de Ética e Pesquisa sob o número 13.203. Resultados: Foram acompanhados 63 pacientes com diagnóstico de neoplasia. Dentre esses, $71,5 \%(n=45)$ estavam em cuidados paliativos. As medianas do tempo de acompanhamento dos pacientes foram de 22 (3-146) dias e do número de visitas realizadas pela equipe foi 6 (1-34). Além das visitas semanais realizadas pelas equipes, os pacientes receberam assistência de nutricionista, fisioterapeuta e assistente social. Considerando as 45 internações, os principais cuidados foram controle da dor, curativos em feridas, controle glicêmico, e antibioticoterapia endovenosa. A principal intercorrência clínica foi a repassagem de cateter nasoenteral $(n=5)$. A alta em condições estáveis de saúde ocorreu em 10 pacientes. Conclusão: $O$ presente estudo permitiu a identificação das principais demandas dos pacientes em cuidados paliativos oncológicos atendidos por um serviço de Atenção Domiciliar. Observa-se na prática deste serviço que a maioria dos pacientes apresentou necessidade de cuidados complexos, mas possíveis de serem realizados no domicílio pela equipe de saúde e/ou cuidador capacitado.

\section{Abstract}

Objective: To identify the profile of palliative care oncology patients and their multidisciplinary needs in a home-care service. Method: A cross-sectional study with secondary data collection from cancer patients in palliative care, attended by the Home-Care Program of Conceição Hospital Group in 2013. Approved by the Ethics and Research Committee under No. 13,203. Results: There were 63 home-care patients diagnosed with cancer. Among those, $71.5 \%(n=45)$ were in palliative care, and $15.5 \%(n=7)$ had more than one admission to the Program. The median follow-up time of all patients was 22 (3-146) days, while for the number of home visits it was 6 (1-34). In addition to weekly home visits, patients were assisted by a nutritionist, a physiotherapist, and a social worker. Concerning the 45 hospitalizations, their main demands were pain management, wound dressings, glycemic control and intravenous antibiotic therapy. The main clinical complication was the reintroduction of a nasoenteral catheter $(n=5)$. Discharge in stable health conditions occurred in 10 patients. Clinical complications and outcomes were analyzed. Conclusion: This study allowed us to identify the main demands of oncological palliative care patients attended by one Home-Care Service. We observed that most patients needed complex care, but the healthcare staff and/or trained caregiver were still able to provide it.
Como citar: Pilatti P, Lagni VB, Picasso MC, Puma K, Mestriner RJS, Machado DO, et al. Cuidados paliativos oncológicos em um serviço público de atenção domiciliar. Rev Bras Med Fam Comunidade. 2017;12(39):1-10. http://dx.doi.org/10.5712/rbmfc12(39)1339

\section{Palavras-chave:}

Cuidados Paliativos

Oncologia

Serviços de Assistência

Domiciliar

Sistema Único de Saúde

\section{Keywords:}

Palliative Care

Medical Oncology

Home-Care Services

Unified Health System
Fonte de financiamento: declaram não haver. Parecer CEP: 13.203 (GHC), aprovado em 13/11/2013.

Conflito de interesses: declaram não haver. Procedência e revisão por pares: revisado por pares. Recebido em: 22/06/2016. Aprovado em: 16/02/2017. 


\section{Resumen}

Objetivo: Identificar el perfil de los pacientes en cuidados paliativos y sus demandas referentes a la atención multidisciplinaria existente en un Servicio de Atención Domiciliaria. Métodos: Estudio transversal con colecta de datos primarios y secundarios de los pacientes oncológicos en cuidados paliativos atendidos por el Programa de Atención Domiciliaria del Grupo Hospitalar Conceição en el año 2013. Aprobado por el Comité de Ética e Investigación (13.203). Resultados: Fueron realizadas 63 internaciones domiciliarias de pacientes con diagnóstico de neoplasia. Entre ellos, un $71,5 \%(n=45)$ estaban en cuidados paliativos, siendo que un $15,5 \%(n=7)$ tuvieron más de un acompañamiento con el Programa. El tiempo promedio de acompañamiento de los pacientes fue de 22 (3-146) días y del número de visitas realizadas por el equipo fue 6 (1-34). Además de las visitas semanales realizadas por los equipos, los pacientes recibieron asistencia con nutricionista, fisioterapeuta y asistente social. Considerando las 45 internaciones, los principales cuidados fueron control del dolor, curación de heridas, control de glucemia, antibioticoterapia intravenosa, entre otros. Fueron analizadas las complicaciones clínicas y los resultados del acompañamiento. Conclusión: Este estudio permitió la identificación de las principales demandas de los pacientes oncológicos en cuidados paliativos atendidos por el servicio de atención domiciliaria. Se observa realizando este servicio que la mayoría de los pacientes necesitó necesidad de atención compleja pero capaz de ser realizado en casa por el equipo de salud y/o cuidador cualificado.
Palabras clave:

Cuidados Paliativos

Oncología Médica

Servicios de Atención de Salud a Domicilio

Sistema Único de Salud

\section{Introdução}

De acordo com a Organização Mundial de Saúde (OMS), o câncer se tornará, em poucas décadas, uma das principais causas de morbidade e mortalidade em todas as regiões do mundo. Estimativas demonstram o aumento da sua incidência de 12,7 milhões em 2008 para 21,4 milhões em 2030. Este fato foi relacionado a diferentes causas, entre elas o aumento da expectativa de vida e o envelhecimento populacional. ${ }^{1}$ Por isso, novas políticas e programas voltados para o tratamento desses indivíduos e para o desenvolvimento e intensificação de estratégias em cuidados paliativos devem ser pautadas pela OMS e implementadas nos diferentes países, sobretudo aqueles que vivem a transição demográfica, como o Brasil.

No Brasil, o Ministério da Saúde estabeleceu a Política Nacional de Atenção Oncológica, que deve ser implantada em todas as unidades federadas e inclui a promoção, prevenção, diagnóstico, tratamento, reabilitação e cuidados paliativos $(\mathrm{CP})$ com garantia de atenção integral aos pacientes, seja através de acompanhamento ambulatorial, hospitalar ou domiciliar. ${ }^{2}$

No que se refere à atenção à saúde dos indivíduos em CP, esta pressupõe uma abordagem multiprofissional que visa à melhoria da qualidade de vida por meio de uma avaliação criteriosa e do tratamento da dor, bem como na atuação diante de outras questões físicas, psicológicas, sociais e espirituais, estendendo-se, inclusive, à fase de luto. ${ }^{3}$ Nesse sentido, a abordagem mutiprofissional se dimensiona em um plano prático, se reconhece no planejamento e na coordenação de ações eficientes para atender de forma integral às necessidades dos pacientes e familiares em articulação com os serviços que compõem a Rede de Atenção a Saúde (RAS). ${ }^{4}$

Dentro desse contexto, caracterizando-se como um serviço de transição do cuidado dentro da RAS, a Atenção Domiciliar (AD) surge como alternativa para esses indivíduos, já que possui um conjunto de ações em saúde tanto de promoção e prevenção, como de tratamento e paliação prestadas em domicílio. ${ }^{5}$ No âmbito do Sistema Único de Saúde (SUS) a Portaria no 2527/2011, por meio do Programa Melhor em Casa (PMC), reorganizou a $A D$ em três modalidades de atendimento conforme a complexidade de cuidado demandada pelos pacientes, prevendo o atendimento domiciliar pela Estratégia de Saúde da Família (ESF) e por serviços específicos de AD. ${ }^{6}$ Os Serviços de AD (SAD) são compostos por equipes multiprofissionais de atenção domiciliar (EMAD) e, também, pelas equipes multiprofissionais de apoio (EMAP). ${ }^{5}$ 
O grande desafio da assistência prestada pelo SAD é compor uma estratégia de cuidados paliativos, visto que se caracteriza como um serviço de transição do cuidado dentro da RAS e que não presta atendimento exclusivo aos pacientes em CP.

Assim, este estudo tem como objetivo identificar o perfil dos pacientes oncológicos em CP e suas demandas referentes ao atendimento multidisciplinar existente em um SAD na região Sul do Brasil com o intuito de analisar, qualificar e/ou renovar as práticas já instaladas.

\section{Métodos}

Trata-se de um estudo transversal de todos os pacientes adultos em cuidados paliativos oncológicos acompanhados pelo Programa de Atenção Domiciliar do Grupo Hospitalar Conceição (PAD-GHC), no ano de 2013. A coleta de dados ocorreu em prontuário eletrônico e em banco de dados institucional.

O diagnóstico de neoplasia foi definido pela Classificação Internacional de Doenças e Problemas Relacionados à Saúde (CID-10) (C00-C97 e D00-D48). ${ }^{7}$ Consideraram-se em cuidados paliativos os pacientes que tinham esta informação presente em nota de alta hospitalar. Foram coletados dados demográficos e referentes ao acompanhamento pelo SAD: número de visitas domiciliares (VD), principais demandas de cuidados, intercorrências e desfechos do acompanhamento. Também foi utilizado um instrumento para avaliação do risco nutricional, o Malnutrition Screening Tool (MUST), que é aplicado na primeira VD e avalia a combinação de estado nutricional atual e da gravidade da doença por meio do índice de massa corporal (IMC), a perda de peso recente e a ingestão alimentar. ${ }^{8}$

A análise estatística foi realizada pelo programa Statistical Package for the Social Sciences (SPSS) 18.0. Os dados foram descritos em média e desvio padrão, mediana e intervalo interquartílico ou frequências.

Este estudo foi aprovado em seus aspectos éticos e metodológicos pelo Comitê de Ética e Pesquisa do Grupo Hospitalar Conceição, sob o parecer no 13.203, e está em conformidade com os princípios contidos na Declaração de Helsinque da Associação Médica Mundial.

\section{Resultados}

Dos 63 (16,9\%) pacientes com diagnóstico de neoplasia no ano de 2013, 45 (71,5\%) estavam em CP. Destes, sete $(15,5 \%)$ tiveram necessidade de mais de um acompanhamento com o serviço no referido ano, ou seja, obtiveram alta do SAD e, posteriormente, foi necessário novo acompanhamento. A média de idade dos pacientes em CP foi de $66( \pm 11,6)$ anos, $60 \%(n=27)$ eram do sexo masculino. A mediana e amplitude do tempo de acompanhamento dos pacientes foi de 22 (3-146) dias e do número de VD realizadas pela equipe de AD foi 6 (1-34).

Além das VD semanais realizadas pelos médicos e equipe de enfermagem, os pacientes receberam assistência de nutricionista, fisioterapeuta e assistente social. As frequências dos principais cuidados, as intercorrências e os desfechos de acompanhamento dos 45 pacientes estão listadas nas Tabelas 1 e 2 , respectivamente. 
Tabela 1. Principais cuidados apresentados pelos pacientes em cuidados paliativos oncológicos acompanhados pelo Programa de Atenção Domiciliar do Grupo Hospitalar Conceição.

\begin{tabular}{lcc}
\hline Variável & $\mathbf{n}$ & $\%$ \\
\hline Cuidados realizados & & 100,0 \\
Controle da dor & 45 & 53,3 \\
Curativos em feridas operatórias, oncológicas ou lesão por pressão & 24 & 33,3 \\
Controle glicêmico & 15 & 31,1 \\
Antibioticoterapia endovenosa & 14 & 17,8 \\
Alimentação por via enteral - manejo de cateteres enterais & 8 & 17,8 \\
Manejo da traqueostomia & 8 & 13,3 \\
Administração de medicamento subcutâneo e intramuscular & 6 & 13,3 \\
Aspiração de vias aéreas ou traqueostomias & 6 & 11,9 \\
Manejo de oxigênio domiciliar & 5 & 11,1 \\
Administração de medicamento por via parenteral & 5 & 8,9 \\
Manejo de jejunostomia e gastrostomia & 4 & 6,7 \\
Manejo de cateter vesical de demora ou intermitente & 3 & \\
\hline
\end{tabular}

Tabela 2. Intercorrências atendidas no domicílio e desfechos do acompanhamento dos pacientes em cuidados paliativos oncológicos atendidos pelo Programa de Atenção Domiciliar do Grupo Hospitalar Conceição.

\begin{tabular}{lcc}
\hline Intercorrências clínicas atendidas no domicílio & $\mathbf{n}$ & $\%$ \\
\hline Exteriorização acidental e repassagem de cateter nasoenteral & 5 & 12,0 \\
Dispneia & 1 & 2,0 \\
Infecção respiratória & 1 & 2,0 \\
Infecção urinária & 1 & 2,0 \\
Dor abdominal & 1 & 2,0 \\
Anemia com necessidade de transfusão sanguínea & 1 & 2,0 \\
\hline Desfechos & & \\
\hline Reinternação hospitalar & 32 & 71,0 \\
Óbito durante a internação hospitalar & 22 & $69,0^{*}$ \\
Alta para acompanhamento na Atenção Básica & 10 & 22,0 \\
Óbito durante o acompanhamento domiciliar & 3 & 7,0 \\
\hline
\end{tabular}

* Dados referentes ao total de pacientes que necessitaram de reinternação hospitalar.

\section{Controle da dor}

Para o manejo da dor, foram utilizados medicamentos (analgésicos opioides, analgésicos não opioides, anti-inflamatórios não esteroides, antidepressivos tricíclicos, entre outros), orientação de técnicas de posicionamento no leito e trocas de postura, assim como exercícios leves de relaxamento e alongamentos. Em casos específicos, foram fornecidas orientações sobre encaminhamentos para acesso a medicamentos que não estão disponíveis na Atenção Primária a Saúde (APS).

\section{Controle glicêmico}

A aferição de glicemia capilar durante as VD possibilitou a otimização do tratamento por meio de orientações dietéticas e medicamentosas (hipoglicemiantes orais e insulina), assim como orientações sobre a aplicação de insulina, com auxílio de manual, e orientações sobre a identificação de sinais de hipoglicemia. 
Curativos em feridas operatórias, oncológicas ou lesões por pressão

Foram encontradas uma ou mais feridas em 24 pacientes, totalizando 33 lesões de pele. As mais prevalentes foram lesão por pressão $(72,7 \%)$, ferida neoplásica $(9,0 \%)$ e ferida operatória $(6,0 \%)$. Foram disponibilizados diferentes tipos de materiais para a realização dos curativos, de acordo com a avaliação e indicação da equipe. Os cuidadores receberam orientações sobre a realização dos curativos durante as VD.

\section{Antibioticoterapia endovenosa}

A utilização de antibioticoterapia por via parenteral no domicílio foi realizada conforme indicação médica e liberação pela comissão de controle de infecção hospitalar. Os medicamentos foram administrados por um técnico de enfermagem sob supervisão de uma enfermeira, uma ou duas vezes ao dia. O acesso venoso foi mantido conforme procedimento operacional padrão para heparinização de cateteres. Foram feitas orientações para os cuidadores e pacientes sobre os cuidados com a manutenção do acesso venoso e possíveis intercorrências.

\section{Alimentação por via enteral - uso de cateteres enterais}

Foi realizada prescrição nutricional individualizada, capacitação sobre a administração, armazenamento de dieta enteral e higienização de materiais, com a disponibilização de manual para consultas. Foi fornecida dieta enteral industrializada para todos os pacientes e encaminhados laudos para solicitação de fornecimento pela Secretaria Estadual de Saúde. Em algumas situações, foi realizada avaliação da capacidade de alimentação via oral e, quando possível, retirada do cateter enteral. Por outro lado, quando a equipe identificou as situações de risco nutricional em pacientes com alimentação por via oral, foi realizado o início da terapia nutricional no domicílio (oral ou enteral). Em casos de retirada acidental de cateter enteral, a equipe realizou a repassagem no domicílio.

\section{Traqueostomia}

Foram realizados e orientados cuidados com higienização e manejo do estoma e cânula de traqueostomia, bem como técnicas de higiene brônquica e aspiração de vias aéreas. Foram disponibilizados manual e vídeo com demonstrações sobre as técnicas, com o objetivo de facilitar a capacitação dos cuidadores. Nos casos de indicação clínica, foi realizado o processo de decanulação da traqueostomia com redução do calibre e períodos de oclusão progressivos.

Além dos principais cuidados, algumas demandas foram identificadas e necessitaram intervenção. Entre elas, inapetência, constipação intestinal, desnutrição (80,9\% dos pacientes estavam em alto risco nutricional, 14,3\% em risco médio), necessidade de suplementação alimentar por via oral, manejo do oxigênio domiciliar de forma mais segura e eficaz, orientações aos direitos do paciente com câncer e da rede de apoio para acompanhamento.

\section{Discussão}

A partir dos resultados apresentados é possível perceber uma alta prevalência de cuidados paliativos nos pacientes oncológicos atendidos no ano 2013 no PAD-GHC. Esse fato pode ser justificado pelo perfil da instituição, que possui uma unidade de internação para cuidados paliativos, acolhe em seus ambulatórios 
os pacientes com menor frequência na demanda do cuidado e encaminha ao SAD os pacientes oncológicos com um quadro mais avançado, muitas em vezes em fase terminal da doença.

A literatura apresenta informações variadas acerca da prevalência de dor, porém sempre com percentuais elevados. O estudo de Reis-Pina et al., ${ }^{9}$ por exemplo, relata que um percentual entre 65 a $80 \%$ dos pacientes com câncer avançado apresentaram queixa de dor. Em nosso estudo, a dor esteve presente na totalidade dos pacientes assistidos e sabe-se que o manejo adequado apresenta uma importante medida de cuidado e conforto para o paciente e os seus familiares. ${ }^{10}$

Para isso, é importante saber a origem da dor, que pode ser nocioceptiva ou neuropática. Alguns estudos de revisão relatam que em torno de $20 \%$ das sensações de dor em pacientes oncológicos tem origem neuropática, sendo que esse valor se eleva para $40 \%$ quando se considera origem combinada (neuropática e nocioceptiva). ${ }^{11,12}$

No PAD-GHC, foram seguidas as recomendações da OMS priorizando a analgesia via oral, o uso regular dos medicamentos, a necessidade de cada paciente e o uso da escala analgésica. Nesse sentido, indo ao encontro com o descrito na literatura, foram utilizados analgésicos não opioides (paracetamol, dipirona, anti-inflamatórios não esteroides), os opioides fracos (codeína, tramadol), os opioides fortes (morfina, metadona) e os adjuvantes (amitriptilina, gabapentina, dexametasona). ${ }^{13,14}$ Em todos os casos, buscou-se o controle da dor total por meio de uma abordagem interdisciplinar com medidas não farmacológicas e farmacológicas.

O cuidado relacionado à troca de curativos apontou a prevalência de feridas operatórias, oncológicas e lesões por pressão. As feridas oncológicas representam grande preocupação pela dificuldade de cicatrização, presença de dor, sangramento, odor intenso e alteração da percepção corporal, o que pode interferir na qualidade de vida. ${ }^{15}$ Cabe ressaltar os controles da dor e odor são questões centrais do cuidado. ${ }^{16}$

Para redução do odor, foi realizada a limpeza da lesão associada ao desbridamento de tecido desvitalizado, quando indicado; uso de cobertura para controle do exsudato; em casos de difícil manejo utilizou-se o metronidazol tópico e antissépticos. Para alívio da dor local e em casos de sangramento, foram aplicadas coberturas atraumáticas.

Os resultados revelaram número elevado de pacientes em risco nutricional. Em pacientes oncológicos há diminuição da tolerância ao tratamento e aproximadamente $20 \%$ das mortes são secundárias à desnutrição. Diversos fatores estão envolvidos neste processo: redução de apetite, dificuldades para mastigar e deglutir alimentos, efeitos colaterais do tratamento e aumento da demanda nutricional provocada pelo crescimento do tumor. Estes fatores são agravados por situações socioeconômicas precárias e hábitos alimentares inadequados. ${ }^{17}$ Neste sentido, a identificação do risco nutricional e intervenções imediatas, como a suplementação por via oral ou passagem de cateteres enterais, fizeram-se necessárias nos pacientes deste estudo.

A intercorrência mais frequente no presente estudo foi a exteriorização acidental e repassagem de cateter nasoenteral, o que poderia ser minimizado com a realização de gastrostomia ou jejunostomia. Porém, a opção de manter o cateter nasoenteral ocorreu, na maior parte das vezes, pelo prognóstico reservado desses pacientes e a tentativa de evitar novas intervenções ambulatoriais que não interfeririam no desfecho. 
Um ponto a ser ressaltado é o fato que grande parte dos pacientes necessitou de reinternação hospitalar e os desfechos demonstraram que a maioria deles foi a óbito, enquanto no domicílio ocorreram apenas três óbitos. O domicílio costuma ser o local de preferência dos pacientes e familiares na fase terminal da doença, ainda que essa decisão possa ser modificada, particularmente, nos últimos dias ou horas que antecedem o óbito. ${ }^{18}$

Uma revisão sistemática conduzida com 31 estudos (amostras entre 42 e 4175 indivíduos), cujo objetivo foi avaliar os determinantes do local da morte em adultos em cuidados paliativos, observou uma ampla variação na taxa de óbito no domicílio, que ficou entre $20 \%$ a $66 \%$. Dentre os estudos que reportaram as preferências de local de morte, $40 \%$ a $85 \%$ dos pacientes preferiu óbito no domicílio, enquanto que $42 \%$ a $65 \%$ dos familiares optaram pelo óbito domiciliar. ${ }^{19}$ Essa ampla variação nas taxas e as diferentes opiniões de pacientes e familiares evidenciam a complexidade do assunto quando o mesmo se coloca em pauta.

Na prática diária, percebe-se que, muitas vezes, os familiares ao presenciarem momentos de disfunção clínica ou de dor, tendem a ficar ansiosos e angustiados, o que dificulta a manutenção da escolha do óbito no domicílio. Nos casos acompanhados pelas equipes do PAD-GHC observa-se que paciente e família não estão totalmente seguros e preparados para enfrentar o momento da morte no domicílio. Outro ponto importante a ser considerado é que, na maioria das vezes, os pacientes em CP recebem alta do hospital para a AD em um momento relativamente próximo do óbito, o que dificulta a formação de um vínculo forte e sensibilização da equipe com os familiares, a ponto de proporcionar segurança e tranquilidade para a tomada de uma decisão complexa como o óbito no domicílio.

Um outro grande desafio a ser enfrentado no atendimento aos pacientes em CP no domicílio, diz respeito ao preparo profissional para lidar e abordar o luto com a família. Entende-se que esse é um tema que precisa ser trabalhado em espaços de discussão e suporte para as próprias equipes. Autores como Pereira (2014) $)^{20}$ destacam a importância da capacitação dos profissionais envolvidos em CP e relacionam o trabalho como "experimentar juntos a dor, o sofrimento, as disputas e tensões entre usuário e familiares, os desejos de cada um, negociar diante dos conflitos, para que se garanta a defesa da vida na iminência da morte".

Conforme Mendes e Vasconcellos (2015), ${ }^{4}$ é necessária uma efetiva mudança do modelo fragmentado de assistência em CP, em que os princípios do SUS sejam garantidos e haja apoio social e psicológico aos pacientes e familiares nesse momento tão delicado em que se encontram, bem como, a interlocução entre essas várias áreas do cuidado. AAD pode potencializar novas formas de produção do cuidado e de atuação atrelada às RAS, com foco nas necessidades de saúde dos pacientes. ${ }^{21}$

Durante o período de acompanhamento do SAD são orientados os direitos dos pacientes com neoplasia e a rede de apoio disponível, podem-se citar algumas experiências exitosas, incluindo alguns serviços de atenção básica ( $A B)$, centros de referência de assistência social (CRAS) e associações como a Associação de Pacientes com Câncer (AAPECAN). A relação com outros serviços deve ser estimulada, principalmente com a $A B$, que é a responsável pela coordenação do cuidado dos pacientes.

Segundo a experiência do PAD-GHC, observa-se que ainda há uma fragmentação da atenção prestada na RAS como um todo, sendo que os serviços não compartilham, entre si e com a família, os 
planos terapêuticos. Dessa forma, existem desafios no que diz respeito a uma atenção com vistas à integralidade do cuidado, tanto em relação ao suporte dos serviços quanto à preparação dos profissionais de saúde para promover o cuidado junto às famílias.

Portanto, promover a qualificação profissional das equipes para trabalhar com AD e, também, com $\mathrm{CP}$, diante da complexidade e necessidade de múltiplos cuidados despendidos por esses pacientes, é extremamente necessário e urgente. Algumas iniciativas de educação permanente já têm sido promovidas em parcerias com a Universidade Aberta do SUS (UNA-SUS) e outras universidades, porém é necessário introduzir e aprofundar esses conteúdos no processo de formação de todos os profissionais da saúde. ${ }^{22}$

$A$ transição do cuidado entre a $A B$ e $A D$ envolve a discussão sobre o momento adequado da transferência dos cuidados entre essas equipes (referência e contrarreferência), assim como sobre a falta de mecanismos reguladores e eficazes de transição que possam potencializar o uso da AD. ${ }^{22}$ Atualmente, o PAD-GHC faz contato com a AB, por meio de telefonemas ou de reuniões, seja para transição do cuidado ou mesmo para que a mesma tenha conhecimento da nova situação do paciente. A falta de recursos humanos, a necessidade de VD mais longas, mais frequentes e não programadas, a não implantação de ESF, a dificuldade de acesso e as situações de violência urbana podem ser apontados como fatores que interferem na efetivação da transição do cuidado. ${ }^{18,23}$

Muitos desafios ainda estão postos para a AD na atualidade, mas ela parece ser uma modalidade de atendimento que pode fortalecer o SUS e a RAS como um todo.

\section{Conclusão}

O presente estudo permitiu a identificação das principais demandas dos pacientes em CP oncológicos atendidos por um serviço de AD. Observa-se na prática deste serviço que a maioria dos pacientes apresentou necessidade de cuidados complexos, mas possíveis de serem realizados no domicílio pela equipe de saúde e/ou cuidador capacitado.

Considerando a complexidade, tanto clínica quanto psicológica, destes pacientes e cuidadores, conclui-se que é necessária a implantação de EMAD e EMAP com competências para lidar com esse contingente populacional. Além disso, a RAS precisa ser articulada e, especificamente, os SAD e a AB necessitam de uma relação estreita e fortalecida para que haja a concretização dos objetivos em prol dos pacientes e um fortalecimento do SUS.

Alguns caminhos para erguer essa ponte entre os SAD e a AB são a construção do plano terapêutico singular, a qualificação profissional focada na RAS desde a formação até a educação permanente e a viabilização de mecanismos reguladores e eficazes de transição do cuidado.

Não há dúvidas de que a AD é uma prática inovadora, considerando-se a transição epidemiológica e demográfica do Brasil. No entanto, sugere-se o desenvolvimento de novas pesquisas para aprofundar as potencialidades da AD em CP, buscando aperfeiçoar a prática existente para que o processo saúde-doença e morte sejam mais dignos, seguros e humanizados. 


\section{Referências}

1. World Health Organization (WHO). Global status report on noncommunicable diseases 2010. Geneva: WHO; 2011.

2. Brasil. Ministério da Saúde. Portaria № 874 de 16 de Maio de 2013. Institui a Política Nacional para a Prevenção e Controle do Câncer na Rede de Atenção à Saúde das Pessoas com Doenças Crônicas no âmbito do Sistema Único de Saúde (SUS). Brasília: Ministério da Saúde; 2013.

3. World Health Organization (WHO). National Cancer control Programmes: Policies and managerial guidelines world. Geneva: WHO; 2002.

4. Mendes EC, Vasconcellos LCF. Cuidados paliativos no câncer e os princípios doutrinários do SUS. Saúde Debate. 2015;39(106):881-92.

5. Brasil. Ministério da Saúde. Portaria № 825 de 25 de abril de 2016. Redefine a Atenção Domiciliar no âmbito do Sistema Único de Saúde (SUS) e atualiza as equipes habilitadas. Brasília: Ministério da Saúde; 2016.

6. Brasil. Ministério da Saúde. Portaria № 2.527 de 27 de Outubro de 2011. Redefine a Atenção Domiciliar no âmbito do Sistema Único de Saúde (SUS). Brasília: Ministério da Saúde; 2011.

7. Organização Mundial da Saúde. CID-10: classificação estatistica internacional de doenças e problemas relacionados a saúde. São Paulo: EDUSP, 1997. p.1191.

8. British Association for Enteral and Parenteral Nutrition. The MUST Explanatory Booklet. A guide to malnutrition universal screening tool (MUST) for adults. Malnutrition Advisory Group (MAG). [acesso 2014 Out 30]. Disponível em: http://www. bapen.org.uk/pdfs/must/must_explan.pdf

9. Reis-Pina P, Lawlor PG, Barbosa A. Cancer-Related Pain Management and the Optimal Use of Opioids. Acta Med Port. 2015;28(3):376-81. DOI: http://dx. doi.org/10.20344/amp.5801

10. Velloso M, Jardim JR. Funcionalidade do paciente com doença pulmonar obstrutiva crônica e técnicas de conservação de energia. J Bras Pneumol. 2006;32(6):580-6. DOI: http://dx.doi.org/10.1590/S1806-37132006000600017

11. García de Paredes ML, del Moral González F, Martínez del Prado P, Martí Ciriquián JL, Enrech Francés S, Cobo Dols $\mathrm{M}$, et al. First evidence of oncologic neuropathic pain prevalence after screening 8615 cancer patients. Results of the On study. Ann Oncol. 2011;22(4):924-30.

12. Roberto A, Deandrea S, Greco MT, Corli O, Negri E, Pizzuto M, et al. Prevalence of Neuropathic Pain in Cancer Patients: Pooled Estimates From a Systematic Review of Published Literature and Results From a Survey Conducted in 50 Italian Palliative Care Centers. J Pain Symptom Manage. 2016;51(6):1091-102.e4. DOI: http://dx.doi.org/10.1016/j. jpainsymman.2015.12.336

13. Mercadante S. Breakthrough pain in cancer patients: prevalence, mechanisms and treatment options. Curr Opin Anaesthesiol. 2015;28(5):559-64. DOI: http://dx.doi.org/10.1097/ACO.0000000000000224

14. Løhre ET, Klepstad P, Bennett MI, Brunelli C, Caraceni A, Fainsinger RL, et al. From "Breakthrough" to "Episodic" Cancer Pain? A European Association for Palliative Care Research Network Expert Delphi Survey Toward a Common Terminology and Classification of Transient Cancer Pain Exacerbations. J Pain Symptom Manage. 2016;51(6):1013-9. DOI: http://dx.doi. org/10.1016/j.jpainsymman.2015.12.329

15. Aguiar RM, Silva GRC. Os cuidados de enfermagem em feridas neoplásicas na assistência paliativa. Rev Hosp Univ Pedro Ernesto. 2012;11(2):82-8.

16. Agreda JJS, Bou JET. Atenção integral nos cuidados das feridas crônicas. Petrópolis: EPUB; 2012.

17. Brasil. Ministério da Saúde. Instituto Nacional de Câncer José de Alencar - INCA. Consenso Nacional de Nutrição Oncológica. Brasília: Ministério da Saúde; 2011.

18. Floriani CA, Schramm FR. Desafios morais e operacionais na inclusão dos cuidados paliativos na rede de atenção básica. Cad Saúde Pública. 2007;23(9):2072-80. DOI: http://dx.doi.org/10.1590/S0102-311X2007000900015

19. Gomes B, Calanzani N, Gysels M, Hall S, Higginson IJ. Heterogeneity and changes in preferences for dying at home: a systematic review. BMC Palliat Care. 2013;12:7. DOI: http://dx.doi.org/10.1186/1472-684X-12-7 
20. Pereira PBA. Atenção domiciliar e produção do cuidado: apostas e desafios atuais [Dissertação de mestrado]. São Paulo: Universidade Federal de São Paulo; 2014.

21. Oliveira Neto AV, Dias MB. Atenção Domiciliar no Sistema Único de Saúde (SUS): o que representou o Programa Melhor em Casa? Divulg Saúde Debate. 2014;51:58-71.

22. Dias MB, Savassi LCM, Nunes MRMTP, Zachi MLR. A Política Nacional de Atenção Domiciliar no Brasil: potencialidades, desafios e a valorização necessária da atenção primária à saúde. J Manag Prim Heal Care. 2015;6(1):1-7.

23. Savassi LCM. Os atuais desafios da Atenção Domiciliar na Atenção Primária à Saúde: uma análise na perspectiva do Sistema Único de Saúde. Rev Bras Med Fam Comunidade. 2016;11(38):1-12. DOI: http://dx.doi.org/10.5712/rbmfc11(38)1259

a Programa de Atenção Domiciliar do Grupo Hospitalar Conceição. Porto Alegre, RS, Brasil. pattipilla@yahoo.com.br; lagni@uol.com.br; raqueljseixas@gmail.com; dianimachado@ hotmail.com; satijaber@gmail.com (Autor correspondente); nanejardim123@yahoo.com.br; heloisa.a.social@gmail.com; karine.personaldiet@gmail.com

b Universidade Federal de Ciências da Saúde de Porto Alegre (UFCSPA). Porto Alegre, RS, Brasil. milenepicasso@ hotmail.com; karenpumall@ hotmail.com; carine.blatt@gmail.com 